\title{
Design of 8-bit SAR-ADC CMOS
}

\begin{abstract}
Successive approximation analog-to-digital converter (ADC) implemented in a conventional $0.18 \varepsilon \mathrm{m}$ CMOS technology with low voltage. The SAR composite of sample-and-hold dummy switch compensation was employed, comparator is low-voltage latched and realized based on current-mode approach, control logic circuit and digital-to-analog conversion consists of binary weighted capacitor arrays for the differential inputs. The ADC has INL and DNL of $0.45 \mathrm{LSB}$ for supply voltage $1.8 \mathrm{~V}$, at sampling rate $200 \mathrm{KS} / \mathrm{S}$ and signal to noise ratio distortion is $58.5 \mathrm{~dB}$. This design is suitable for standard CMOS technology with lowpower low-cost VLSI implementation. It is well applied when embedded into system-on-chip (SOC) circuit designs.
\end{abstract}

Keyword: Analog-to-digital converter; CMOS; Comparator; Digital-to-analog converter; Low voltage; Sample-and-hold 